\title{
Correction to: Tensor Algebra
}

\section{Correction to:}

Chapter 1 in: A. Romano and M. Mango Furnari, The Physical and Mathematical Foundations of the Theory of Relativity, https://doi.org/10.1007/978-3-030-27237-1_1

Initially the book was published without the Extra Supplementary Material. Now it has been included in chapter 1, titled "Tensor Algebra". 